\title{
Pseudotumoral actinomycotic mycetoma of the buttock in children: A case report
}

\section{Khadim Diop, Boubacar Ahy Diatta, Saer Diadie, Niare Ndour, Coumba Ndiaye, Aminata Deh, Maodo Ndiaye, Moussa Diallo, Suzanne Oumou Niang}

Service de Dermatologie, Hôpital Aristide Ledantec, Dakar, Senegal

Corresponding author: Khadim Diop, MD, E-mail: bambadiop100391@gmail.com

\begin{abstract}
ABSRACT
Mycetoma is a rare and neglected tropical disease among children. Extrapodal forms, in particular the buttock, are serious because they expose to visceral extension. We are reporting an observation remarkable for its clinical tumor presentation in a child. An 11 -year-old boy was admitted at to the dermatology department for a buttock tumor that has been evolving for 2 years. Physical examination showed a multinodular, polyfistulized, and inflammatory tumor emitting red granules located in the buttocks. Mycological examination isolated Actinomadure pelletieri that confirms the diagnosis of actinomicotic mycetoma. The evolution, after a two-year follow-up, was positive under antibiotic therapy combining cotrimoxazol, clavulanic acid amoxicillin and streptomycin. Our observation is specific by the occurrence of a pseudotumoral actinomycotic buttock mycetoma in a child, the diagnostic delay and the favorable evolution without recurrence after a follow-up of 24-month.
\end{abstract}

Key words: Actinomycotic mycetoma; Buttock; Child

How to cite this article: Diop K, Diatta BA, Diadie S, Ndour N, Ndiaye C, Deh A, Ndiaye M, Diallo M, Niang SO. Pseudotumoral actinomycotic mycetoma of the buttock in children: A case report. Our Dermatol Online. 2021;12(Supp. 1):21-25.

Submission: 25.11.2020; Acceptance: 01.01.2021

DOI:10.7241/ourd.2021s1.5 


\section{Un mycétome actinomycosique pseudotumoral de la fesse chez l'enfant : une observation \\ Khadim Diop, Boubacar Ahy Diatta, Saer Diadie, Niare Ndour, Coumba Ndiaye, Aminata Deh, Maodo Ndiaye, Moussa Diallo, Suzanne Oumou Niang}

Service de Dermatologie, Hôpital Aristide Ledantec, Dakar, Senegal

Corresponding author: Khadim Diop, MD, E-mail: bambadiop100391@gmail.com

RÉSUMÉ

Le mycétome est une maladie tropicale négligée rare chez l'enfant. Les formes extrapodales en particulier fessière sont graves car exposant au risque d'extension viscérale. Nous en rapportons une observation chez un enfant remarquable par sa présentation clinique tumorale. Un garçon âgé de 11 ans, était admis au service de Dermatologie, pour une tumeur fessière évoluant depuis 2 ans. Lexamen physique montrait une tumeur, multinodulaire, polyfistulisée, inflammatoire, émettant des grains rouges, siégeant au niveau des fesses. Lexamen mycologique a permis d'isoler Actinomadurae Pelletieri confirmant ainsi le diagnostic de mycétome actinomycosique. Lévolution, après un recul de deux ans, était favorable sous antibiothérapie associant le cotrimoxazole, l'amoxicilline acide clavulanique et la streptomycine. Notre observation est particulière par la survenue d'un mycétome pseudotumoral actinomycosique fessier chez un enfant, le retard diagnostic et l'évolution favorable sans récidive après un recul de 24 mois.

Mots-clés: Mycétome actinomycosique; Fesse; Enfant 


\section{INTRODUCTION}

Les mycétomes sont des maladies tropicales négligées endémiques au Sénégal et atteignent très souvent les adultes jeunes entre 20 et 40 ans, avec une localisation podale plus fréquente [1]. La survenue de mycétome chez l'enfant est rarement rapportée dans la littérature médicale. La topographie fessière est rare et représente $0,3 \%$ des cas chez l'enfant [2]. La gravité est liée au risque d'extension locorégionale, pouvant engager le pronostic fonctionnel par l'atteinte osseuse et le pronostic vital par les atteintes viscérales [3]. Nous en rapportons une observation chez un enfant remarquable par sa présentation clinique tumorale.

\section{Observation}

Un garçon âgé de 1 lans, originaire de la région de Matam (450 km de Dakar) était reçu en consultation au service de Dermatologie de l'hôpital Aristide LeDantec pour une tumeur fessière évoluant depuis 2 ans, après des consultations aux services de pédiatrie puis en neurochirurgie. Il avait reçu des traitements traditionnels pendant 18 mois sans amélioration. Lenfant vivait avec ses parents dans une famille où l'activité principale était agropastorale. Linterrogatoire ne rapportait pas de notion de traumatisme inoculateur. A l'admission, l'état général était conservé, la température était à $36,8^{\circ} \mathrm{C}$, la tension artérielle à $120 / 80 \mathrm{mmHg}$, le poids à $33 \mathrm{~kg}$ et la taille à $1,62 \mathrm{~m}$. Lexamen physique objectivait une tumeur multinodulaire, polyfistulisée, inflammatoire, émettant des grains rouges, mesurant $18 \mathrm{~cm}$ de grand axe, siégeant sur les fesses prédominant à gauche s'étendant sur la région sacrale (Fig. 1). Il n'y avait pas d'adénopathies périphériques, ni de signes neurologiques et le reste de l'examen physique était normal. Le prélèvement mycologique mettait en évidence à l'examen direct des grains rouges et la culture sur milieu de Lowenstein Jensen avait permis d'isoler Actinomadura pelletieri (Figs. 2 et 3). Le diagnostic de mycétome actinomycosique était retenu devant l'origine géographique du malade, la tumeur multinodulaire polyfistulisée émettant des grains rouges et l'isolement d'Actinomadurae pelletieri à la culture. Le scanner lombo-sacré et fessier montrait une masse sous cutané tissulaire hétérogéne de $18 \mathrm{~cm}$ de grand axe s'étendant du coccyx à la région lombaire $\left(\mathrm{L}_{3}\right)$ sans envahissement osseux. Léchographie abdominopelvienne était normale. Sur le plan biologique, on notait un syndrome inflammatoire biologique non spécifique (anémie à $10 \mathrm{~g} / \mathrm{dl}$ et une

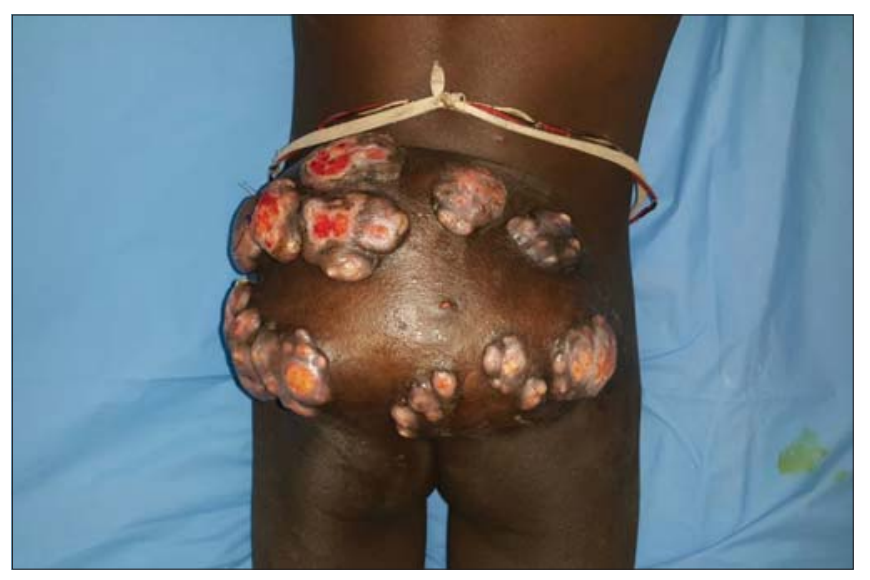

Figure 1: Multinodular polyfistulized tumor of the buttock.

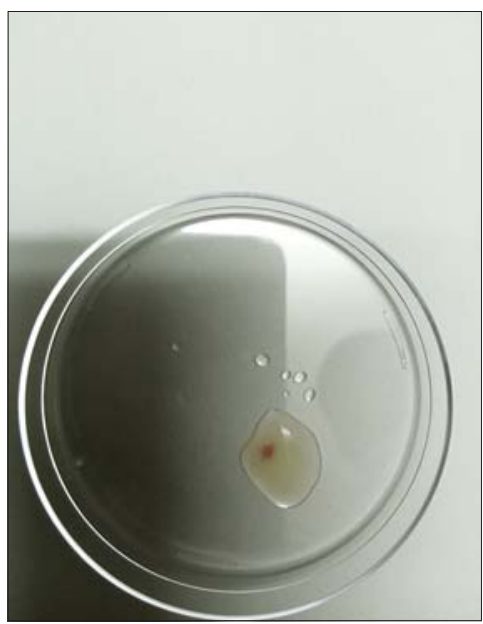

Figure 2: Macroscopy: Presence of red granules.

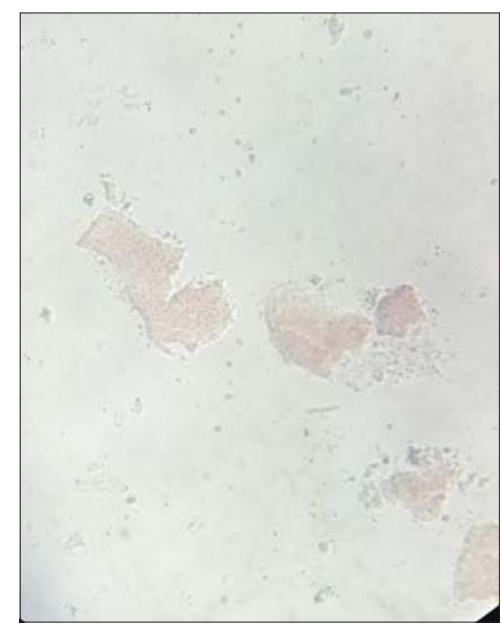

Figure 3: Microscopy: Grouping in clusters of actinomycetes.

hyperleucocytose à $10500 / \mathrm{mm}^{3}$ ). La sérologie rétrovirale était négative. La fonction rénale, l'ionogramme sanguin, la glycémie à jeûn et les transaminases étaient normaux. Le patient était hospitalisé et avait reçu 
comme traitement de la streptomycine injectable à la posologie de $15 \mathrm{mg} / \mathrm{kg} /$ jour pendant 1 mois, associée à du cotrimoxazole $40 \mathrm{mg} /$ jour et de l'Amoxicilline acide clavulanique $40 \mathrm{mg} / \mathrm{kg} /$ jour par voie orale pendant 12 mois avec une surveillance régulière de la fonction rénale, des transaminases et de la fonction auditive. Lévolution clinique était favorable, marquée par un affaissement de la tumeur, une fermeture complète des fistules et des cicatrices atrophiques, sans récidive, après un recul de deux ans (Fig. 4). Le scanner de contrôle montrait un affaissement de la masse sous cutané.

\section{DISCUSSION}

Notre observation est particulière par la présentation clinique tumorale d'un mycétome actinomycosique chez un enfant, la topographie fessière, le diagnostic tardif et l'évolution favorable sans récidive sous triple antibiothérapie.

Le mycétome de l'enfant est rarement rapporté dans la littérature médicale, avec une incidence entre 3 à 4,5\% [2] . Sur le plan épidémiologique, le mycétome chez l'enfant survient en majorité chez les garçons d'âge moyen de 13 ans, et d'origine actinomycosique dans $21,9 \%$ des cas $[2,4]$. La faible incidence du mycétome chez l'enfant pourrait s'expliquer par une exposition moindre aux facteurs de risque. Lorigine géographique du malade, les activités agropastorales de la famille et la fréquentation précoce des champs agricoles pourrait constituer un facteur de risque d'inoculation de l'agent pathogène.

Le caractère tumoral du mycétome prête souvent à confusion avec les tumeurs des tissus mous rendant compte ainsi de l'errance diagnostique observé chez notre malade $[5,6]$. Le Sénégal est une zone d'endémie aux mycétomes. Lexistence de pseudotumeur avec une émission de grains doivent orienter vers un diagnostic de mycétome confirmé par l'examen mycologique, l'histologie ou récemment par la PCR.

Le retard diagnostique constaté chez notre malade attesté par la longue durée d'évolution, pourrait s'expliquer par les difficultés d'accès aux soins spécialisés en Dermatologie ainsi que le plateau technique limité dans les régions $[1,6,7]$. Ce qui explique la consultation chez les tradipraticiens en première intention. Lorientation vers la médecine moderne se fait après échec du traitement traditionnel ou en cas de douleur ou de tuméfaction invalidante [5].

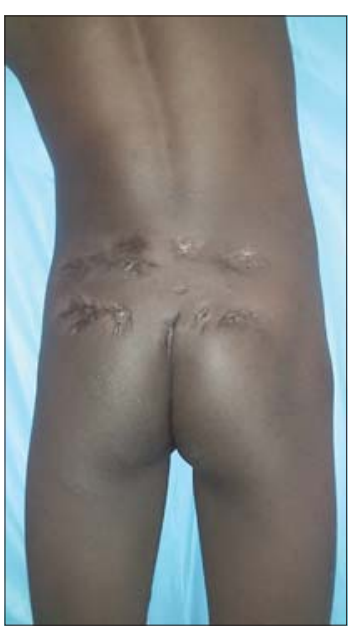

Figure 4 : Atrophic scars after treatment.

La localisation podale des mycétomes est de loin la plus fréquente, environ 81 à $90 \%$ des cas dans les séries africaines $[1,8,9]$. Au Sénégal, Dieng notait que les mycétomes extrapodaux ne représentaient que 38,5\% des mycétomes [3]. La localisation du mycétome au niveau de la fesse est relativement rare. Au Soudan, sur une série de 722 cas de mycétomes chez des enfants, la topographie fessière était retrouvée dans $0,3 \%$ des cas [2]. La rareté de cette topographie pourrait s'expliquer par une moindre exposition aux traumatismes inoculateurs.

La topographie fessière du mycétome est grave car pouvant exposer à un risque d'envahissement du périnée, d'atteintes osseuses vertébrales et du coccyx, menaçant le pronostic fonctionnel par la compression médullaire, les troubles sphinctériens, la réduction de la mobilité et le pronostic vital par les atteintes viscérales. La tomodensitométrie permet la détection des lésions osseuses débutantes et une cartographie des atteintes.

Le Cotrimoxazole, seul pendant 12 mois, constitue le traitement de référence des mycétomes actinomycosiques $[10,11]$. Cependant l'association cotrimoxazole, Streptomycine et amoxicilline acide clavulanique semble être plus efficace en cas de mycétomes pseudotumorales comme l'atteste le cas de ce garçon [5]. Le traitement instauré en hospitalisation était constitué par la Streptomycine à la posologie de $15 \mathrm{mg} / \mathrm{kg} /$ jour en injectable pendant 01 mois. Il était associé à un traitement par voie orale composé de l'amoxicilline acide clavulanique $40 \mathrm{mg} / \mathrm{kg} /$ jour et le cotrimoxazole $40 \mathrm{mg} /$ jour pendant 12 mois. Des complications à type de cytolyse hépatique, de néphrotoxicité et d'ototoxicité pourrait découler de la prise de la streptomycine d'où l'intérêt d'une 
surveillance régulière des transaminases, de la fonction rénale et de la fonction auditive. L'évolution était favorable, après un recul de 2 ans, marqué par un affaissement de la tumeur, une fermeture des fistules et une régression de la tumeur au scanner de contrôle.

\section{CONCLUSION}

Nous rapportons une observation de mycétome actinomycosique remarquable par sa présentation clinique tumorale chez un enfant, de topographie fessière et l'évolution favorable sans récidive sous triple antibiothérapie.

\section{Consent}

The examination of the patient was conducted according to the principles of the Declaration of Helsinki.

The authors certify that they have obtained all appropriate patient consent forms, in which the patients gave their consent for images and other clinical information to be included in the journal. The patients understand that their names and initials will not be published and due effort will be made to conceal their identity, but that anonymity cannot be guaranteed.

\section{RÉFÉRENCES}

1. Ndiaye D, Ndiaye M, Sène PD, Diouf MN, Diallo M, Faye B, et al. Mycetomes diagnostiqués au Sénégal de 2008 à 2010. J Mycol Med. 2011;21:173-81.
2. Fahal AH, Sabaa AA. Mycetoma in children in Sudan. TransR Soc Trop Med Hyg. 2010;104:117-21.

3. Dieng MT, Niang SO, Sy T, Ndir MH, Ndiaye B. Mycétomes extrapodaux : à propos de 50 cas sénégalais. Nouv Dermatol. 2001;20:384-6.

4. Bonifaz A, Ibarra G, SaúL A, Paredes-Solis V, Carrasco-Gerard E, Fierro-Arias L. Mycetoma in children: experience with 15 cases. Pediatr Infect Dis J. 2007;26:50-2.

5. Ndiaye M, Diatta BA, Sow D, Diallo M, Diop A, Diadie S, et al. Une présentation atypique tumorale d'un mycétome actinomycosique dorsolombaire, J Mycol Méd. 2014;24:44-7.

6. Diatta BA, Ndiaye M, Sarr L, Diatta BJM, Gueye AB, Diop A, et al, Un mycétome fongique tumoral dorsal : intérêt de la chirurgie large associée à la terbinafine, J Mycol Med. 2014;24:351-4.

7. Develoux M, Dieng MT, Ndiaye B. Mycétomes. In: EMC - Maladies infectieuses. Paris: Elsevier Masson SAS; 2002: 10_1 [8-606-A].

8. Ndiaye B, Develoux M, Dieng MT, Kane A, Ndir O, Raphenon G, et al. Aspects actuels des mycétomes au Sénégal. À propos de 109 cas. J Mycol Med. 2000;10:140-4.

9. Fuentes-Nava AG, Fierro-Arias L, Araiza J, Benitez-Barradas MI, Peláez González HE, Bonifaz A. Actinomycetoma due to Actinomadura madurae: A therapeutic challenge. Case report. Our Dermatol Online. 2018;9:399-403.

10. Develoux M, Dieng MT, Kane A, Ndiaye B. Prise en charge des mycétomes en Afrique de l'ouest. Bull Soc Pathol Exot. 2003;96:376-82.

11. Salissou L, Mamadou S, Sani R, Hassan N. Giant cervico-facial mycetoma caused by Streptomyces somaliensis in a 14-year-old girl. Our Dermatol Online. 2016;7:62-5.

Copyright by Khadim Diop, et al. This is an open access article distributed under the terms of the Creative Commons Attribution License, which permits unrestricted use, distribution, and reproduction in any medium, provided the original author and source are credited.

Source of Support: Nil, Conflict of Interest: None declared. 